\title{
Comparación del dolor laringotraqueal postoperatorio en adultos sometidos a intubación orotraqueal para cirugía electiva con el uso de bencidamina, lidocaína alcalinizada y placebo, solas o en combinación: Ensayo clínico aleatorizado
}

\author{
Comparison between postoperative sore throat in adults subjected to orotracheal \\ intubation for elective surgery with the use of benzydamine, alkalinized \\ lidocaine and placebo, alone or combined. Randomized clinical trial
}

Lorena Bobadilla Suárez, * Bernardo José Gutiérrez Sougarret, José Manuel Portela Ortiz, ${ }^{\ddagger}$ Luis Antonio García Hernández, ${ }^{\ddagger}$ Ailyn Cendejas Schotman, Jonathan Jair Mendoza Reyes, ${ }^{\ddagger}$ Gabriela Garza Benavides*

Citar como: Bobadilla SL, Gutiérrez SBJ, Portela OJM, García HLA, Cendejas SA, Mendoza RJJ et al. Comparación del dolor laringotraqueal postoperatorio en adultos sometidos a intubación orotraqueal para cirugía electiva con el uso de bencidamina, lidocaína alcalinizada y placebo, solas o en combinación: Ensayo clínico aleatorizado. Acta Med. 2021; 19 (1): 86-91. https://dx.doi.org/10.35366/98576

\section{Resumen}

Introducción: Se ha observado que el uso de bencidamina en aerosol o de lidocaína alcalinizada instilada dentro del globo del tubo endotraqueal es útil para disminuir la incidencia y la intensidad del dolor laringotraqueal postoperatorio. Hasta el momento no se sabe si el uso aislado o asociado de estos fármacos tiene alguna ventaja para reducirlas. Material y métodos: Se realizó un ensayo clínico controlado aleatorizado triple ciego formando cuatro grupos: control (placebo-placebo), lidocaína-bencidamina, placebo-bencidamina, lidocaína-placebo, a los cuales se les asignaron aleatoriamente 18 pacientes por grupo. Se midió la intensidad del dolor mediante una escala numérica análoga a los 15 min, 1 h, 3 h, 12 h y 24 h en cada uno de los grupos, además de otras variables de interés. Resultados: Se analizaron los datos de 72 pacientes y se detectó una diferencia estadísticamente significativa en la intensidad del dolor laringotraqueal en el curso de las primeras 24 horas a favor de uno de los grupos (lidocaínaplacebo $p=0.003)$. Conclusión: El uso de la lidocaína alcalinizada instilada dentro del globo del tubo endotraqueal es más efectivo para disminuir la incidencia e intensidad de dolor laringotraqueal postoperatorio que el uso de la bencidamina sola o combinada.

\section{Abstract}

Introduction: There have been promising results for decreasing the incidence and intensity of postoperative sore throat with the use of sprayed benzydamine or intracuff alkalinized lidocaine. At the time of our review, there were no reports that found a superior outcome of their use either alone or as adjuncts. Material and methods: We conducted a triple blinded randomized controlled trial composed of four groups: control (placebo-placebo), lidocaine-benzydamine, placebobenzydamine, lidocaine-placebo with 18 patients randomly allocated to each group. We recorded NAS (numerical analog scale) at $15 \mathrm{~min}, 1 \mathrm{~h}, 3 \mathrm{~h}, 12 \mathrm{~h}$, and $24 \mathrm{~h}$ for each group as well as other variables of interest. Results: We analyzed the data from 72 patients and detected a statistically significant difference in the intensity of sore throat during the first 24 hours in favor of one group (lidocaine-placebo with a $p=0.003$ ). Conclusion: Intracuff alkalinized lidocaine is more effective in decreasing the incidence and intensity of postoperative sore throat compared to the use of benzydamine alone or as an adjunct to alkalinized lidocaine.
* Residente de Anestesiología. Facultad Mexicana de Medicina de la Universidad La Salle.

‡ Departamento de Anestesiología.

\section{Correspondencia:}

Lorena Bobadilla Suárez

Correo electrónico: dra.lorena.bobadilla@gmail.com

Aceptado: 09-11-2020.

Hospital Ángeles Pedregal. Ciudad de México, México.

www.medigraphic.com/actamedica 
Palabras clave: Dolor laringotraqueal postoperatorio, lidocaína alcalinizada, bencidamina, intubación orotraqueal, ronquera.

\section{INTRODUCCIÓN}

El efecto adverso más frecuente después de la intubación orotraqueal es el dolor laringotraqueal con una incidencia que varía según la literatura desde 15 hasta 96\%. ${ }^{1-11}$ Entre los factores de riesgo de desarrollar el mismo se encuentran el género femenino, la dificultad durante la intubación, la edad mayor de 60 años, el uso de tubos endotraqueales grandes y el inflado excesivo o aumento de la presión del globo del tubo endotraqueal. $8,9,11,12$

Se cree que el dolor laringotraqueal se presenta principalmente por irritación local causada por el inflado excesivo del globo, lo que puede impedir el adecuado flujo sanguíneo en los capilares de la mucosa traqueal ${ }^{13}$ o por resequedad de la vía aérea debido al uso de gases fríos y secos, lo cual provoca adherencia del tubo a las paredes de la tráquea.

Se han propuesto múltiples intervenciones para disminuir tanto la incidencia como la intensidad del dolor laringotraqueal posterior a la intubación, 5,, 12 que va desde el uso de lidocaína o bencidamina en aerosol en el exterior del tubo o directamente en la faringe hasta la instilación de lidocaína dentro del tubo endotraqueal, ${ }^{1-7,10,12-15}$ pero aún no se ha dilucidado cuál maniobra es la más efectiva ni se han comparado las maniobras entre sí., ${ }^{3,4}$

El dolor laringotraqueal está asociado a la insatisfacción del paciente y a que considere su anestesia como una mala experiencia; ;,9,10,16 de ahí la importancia de tratar de paliar en lo posible la molestia para mejorar
Keywords: Postoperative sore throat, alkalinized lidocaine, benzydamine, orotracheal intubation, hoarseness. la satisfacción del paciente en el postoperatorio inmediato. $1,7,11,12,17$

Después de una extensa revisión de la literatura, establecimos como objetivo la comparación de lidocaína alcalinizada y bencidamina para evaluar su efecto en la incidencia e intensidad del dolor laringotraqueal en pacientes que reciban anestesia general con intubación orotraqueal en cirugía electiva.

\section{MATERIAL Y MÉTODOS}

Se realizó un ensayo clínico controlado doble ciego autorizado por el comité de ética del Hospital Ángeles Pedregal, para lo cual se crearon cuatro grupos: el grupo PP (placebo-placebo) con solución estéril infundida dentro del globo del tubo endotraqueal más solución estéril por fuera del mismo; el grupo LB (lidocaína-bencidamina) con lidocaína alcalinizada (160 mg de lidocaína simple al 2\% alcalinizada más $2 \mathrm{~mL}$ de bicarbonato de sodio al 7.5\%) infundida dentro del globo del tubo endotraqueal más bencidamina $(1.5 \mathrm{mg} / \mathrm{mL})$ por fuera del globo del tubo endotraqueal; el grupo PB (placebo-bencidamina) con solución estéril dentro del globo del tubo endotraqueal más bencidamina por fuera del globo del tubo endotraqueal; y LP (lidocaína-placebo) con lidocaína al 2\% alcalinizada por dentro del globo del tubo endotraqueal y solución estéril por fuera. En todos los grupos se infundió la cantidad necesaria de la solución asignada para evitar la fuga de aire con una presión de $30 \mathrm{CmH}_{2} \mathrm{O}$ y cuatro disparos de

Tabla 1: Características demográficas de los pacientes estudiados.

\begin{tabular}{|c|c|c|c|c|c|}
\hline & \multicolumn{5}{|c|}{ Grupos experimentales } \\
\hline & PP & LB & PB & LP & $p$ \\
\hline Edad (años) & $46.5(36.8-65.5)$ & $47(34.5-57.5)$ & $43(35-56.8)$ & $50(31-62.5)$ & 0.84 \\
\hline Peso (kg) & $74.5(68-91.3)$ & $74(60.5-81)$ & 75.5 (64.8-82.8) & $70(61-75.5)$ & 0.52 \\
\hline Talla (m) & $1.7(1.63-1.76)$ & $1.67(1.63-1.75)$ & $1.63(1.6-1.7)$ & $1.65(1.62-1.7)$ & 0.41 \\
\hline $\mathrm{IMC}\left(\mathrm{kg} / \mathrm{m}^{2}\right)$ & $27.0(24.3-29.6)$ & 25.5 (21.1-28.7) & $26.0(24.2-31.8)$ & $24.1(23.5-27.1)$ & 0.49 \\
\hline Género F/M (\%) & $38 / 63$ & $47 / 53$ & $50 / 50$ & $53 / 47$ & 0.83 \\
\hline ASA I/II/III (\%) & $44 / 44 / 12$ & $47 / 33 / 20$ & $37 / 44 / 19$ & $47 / 40 / 13$ & 0.98 \\
\hline
\end{tabular}


Tabla 2: Características de la vía aérea e intubación.

\begin{tabular}{|c|c|c|c|c|c|}
\hline & \multicolumn{5}{|c|}{ Grupos experimentales } \\
\hline & PP & LB & PB & LP & $p$ \\
\hline Mallampati I/II/III (\%) & $44 / 50 / 6$ & $46 / 27 / 27$ & $37 / 38 / 25$ & $47 / 33 / 20$ & 0.74 \\
\hline Apertura bucal I/II/III (\%) & $75 / 19 / 6$ & $53 / 33 / 12$ & $50 / 44 / 6$ & $87 / 13 / 0$ & 0.27 \\
\hline Patil-Aldreti I/II/III (\%) & $63 / 31 / 6$ & $53 / 40 / 7$ & $31 / 56 / 13$ & $60 / 40 / 0$ & 0.63 \\
\hline IPID (puntos) & $5(4-6.25)$ & $5(4-7)$ & $6.5(4.75-7.25)$ & $5(4-6)$ & 0.29 \\
\hline IPID grado de dificultad F/D (\%) & $94 / 6$ & $80 / 20$ & $75 / 25$ & $93 / 7$ & 0.33 \\
\hline Tipo de laringoscopia D/V (\%) & $94 / 6$ & $100 / 0$ & $80 / 20$ & $100 / 0$ & 0.78 \\
\hline Hoja Macintosh 3/4 (\%) & $56 / 44$ & $64 / 36$ & $67 / 33$ & $64 / 36$ & 0.39 \\
\hline Uso de guía S/N (\%) & $94 / 6$ & $57 / 43$ & $73 / 27$ & $64 / 36$ & 0.91 \\
\hline Número de intentos de intubación 1/2/3 (\%) & $87 / 13 / 0$ & $79 / 14 / 7$ & $80 / 7 / 13$ & $79 / 21 / 0$ & 0.72 \\
\hline Número de tubo endotraqueal 7.0/7.5/8.0 (\%) & $31 / 6 / 63$ & $50 / 0 / 50$ & $53 / 0 / 47$ & $57 / 7 / 36$ & 0.72 \\
\hline Cormack I/II/II/IV (\%) & $50 / 25 / 19 / 6$ & $71 / 0 / 21 / 7$ & $40 / 20 / 40 / 0$ & $64 / 22 / 7 / 7$ & 0.68 \\
\hline Experiencia del laringoscopista I/II/III/AD (\%) & $19 / 13 / 62 / 6$ & $21 / 21 / 58 / 0$ & $7 / 13 / 67 / 13$ & $14 / 29 / 50 / 7$ & 0.68 \\
\hline
\end{tabular}

la solución asignada por fuera del tubo endotraqueal. La asignación del grupo a cada paciente se realizó a través de sobres sellados previamente numerados hasta 144 que contenían el tratamiento asignado de manera aleatoria; la aleatorización se hizo en bloques de cuatro y el orden de los tratamientos se asignó mediante el uso de una tabla de números aleatorios, de tal forma que para cuatro pacientes se emplearon los cuatro tratamientos propuestos (PP, LB, PB y LP). Una vez que el paciente aceptaba participar en el estudio y habiendo firmado el consentimiento informado, el personal técnico de anestesia procedía a abrir el sobre que le correspondía al paciente y preparaba el tubo endotraqueal sin que el anestesiólogo a cargo del caso tuviera conocimiento del tratamiento del paciente. El técnico de anestesia desconocía la hipótesis en estudio y no estuvo involucrado en la evaluación del dolor postoperatorio de los pacientes.

Para una diferencia de dos unidades en la ENA (escala numérica análoga) entre el grupo control (globo lleno con solución inyectable y agua inyectable tópica a nivel del globo) y cualquiera de los grupos experimentales (LB, PB, LP) en cualquiera de las evaluaciones postoperatorias con una probabilidad de cometer un error tipo I (error $\alpha$ ) de 0.001 y una probabilidad de cometer un error tipo II (error $1-\beta)$ de 0.9 se necesitarían 36 pacientes en cada grupo con un total de 144 pacientes.

Los criterios de inclusión fueron pacientes ASA I-III, mayores de 18 años, programados para cirugía electiva que re- quirieran anestesia general con intubación orotraqueal. Se excluyeron procedimientos que involucraban manipulación de laringe, o faringe y pacientes con síntomas respiratorios previo al procedimiento. Como criterio de eliminación de estudio se contempló la posibilidad de que una vez aleatorizado el paciente se hubiera decidido de último momento una técnica anestésica sin intubación orotraqueal o que por complicaciones durante el procedimiento no se registraran todas las variables de forma adecuada.

Se registraron los datos demográficos más importantes de cada paciente (edad, género, peso, talla, comorbilidades y diagnósticos) así como el tipo de cirugía. Además, se registraron datos como la duración de la cirugía, la duración de la anestesia, el tiempo transcurrido entre el término de la anestesia y la extubación del paciente, la dosis total de narcótico (expresada en equivalencia de dosis total de fentanilo), uso de analgésicos, tos y náusea a la extubación así como la presencia de dolor laringotraqueal a los 15 min, 1 h, 3 h, 12 h y 24 h. Adicionalmente se evaluaron diversos índices de dificultad para el manejo de la vía aérea.

Se utilizó la escala numérica análoga (ENA) para valorar dolor laringotraqueal, donde 0 es ausencia de dolor y 10 es el dolor más intenso que el paciente pueda imaginar. Para evitar sesgos al momento de evaluar el dolor se inició la evaluación preguntando si el paciente tenía alguna molestia, posteriormente si sentía dolor en alguna parte del cuerpo y por último si presentaba dolor laringotraqueal, 
de tal forma que el paciente fuera lo más objetivo posible durante la evaluación del dolor que sentía. 9,15

El análisis estadístico fue realizado por uno de los autores (BJGS), quien no participó en la preparación de tubos endotraqueales ni en la valoración del dolor postoperatorio. El análisis estadístico interim (cuando se alcanzó la mitad del tamaño de la muestra) se hizo sin tener conocimiento del grupo al que pertenecía cada paciente (triple ciego).

Para describir las variables se utilizaron medidas de tendencia central y dispersión (media, mediana, desviación típica, intervalo intercuartil y porcentajes), dependiendo de la distribución de los datos analizados y pruebas de $t$ de Student, $U$ de Mann-Whitney, $\chi^{2}$ o prueba exacta de Fisher para evaluar diferencias entre las variables medidas. Por ultimo, se hizo un análisis multivariado con pruebas de ANDEVA (análisis de varianza) de dos y tres vías. Se consideró como estadísticamente significativo un valor de $\mathrm{p}<0.05$. Al realizar el análisis interim se encontró una diferencia estadísticamente significativa, por lo que se decidió develar el ciego y dar por terminado el estudio, ya que fue financiado por los mismos investigadores y así no incurrir en gastos adicionales. Al haber calculado la muestra con parámetros conservadores ( $\alpha$ de 0.001 y $1-\beta$ de 0.9 ) consideramos completar la muestra sólo si no existían resultados claros al realizar el análisis preliminar. Con parámetros más liberales (p. ej. $\alpha$ de 0.05 y 1- $\beta$ de 0.8) hubiera resultado deseable completar la muestra estimada de 144 pacientes.

\section{RESULTADOS}

Se analizaron los datos de 72 pacientes. Las características demográficas de la muestra estudiada se observan en la Tabla 1 ; las diferencias existentes entre los distintos grupos no fueron estadísticamente significativas. Se evaluaron diversas variables que pudieran influir en la aparición y la intensidad de dolor laringotraqueal postoperatorio de forma previa, durante y después de la intubación; asimismo se midieron diversos índices para clasificar la dificultad de la vía aérea; no se detectaron diferencias estadísticamente significativas entre los grupos experimentales (Tablas 2 y 3). Se observó que la frecuencia de dolor laringotraqueal de cualquier intensidad es baja, de $22.4 \%$ a las 3 horas la más alta (Figura 1). La frecuencia de ENA mayor a 3 fue, de igual forma, la más alta a las 3 horas, con un $10.3 \%$ (Figura 2). Al realizarse el ANDEVA de dos factores se encontró que el ENA era significativamente menor en el grupo IV (LP) a las 3, 12 y $24 \mathrm{~h}$, seguido del grupo III (PB) de igual forma a las 3, 12 y $24 \mathrm{~h}$ (Figura 3). No existieron diferencias significativas entre los cuatro grupos a los 15 min y $1 \mathrm{~h}$ del postoperatorio, pero sí se observó menor frecuencia e intensidad en el grupo II (LB) en ambos momentos.

\section{DISCUSIÓN}

La presencia de dolor laringotraqueal en nuestra población resultó ser mucho más baja que lo esperado, siendo de $22.4 \%$ su frecuencia máxima, tomando en consideración

Tabla 3: Características de anestesia, cirugía, extubación y analgesia postoperatoria.

\begin{tabular}{|c|c|c|c|c|c|}
\hline & \multicolumn{5}{|c|}{ Grupos experimentales } \\
\hline & PP & LB & PB & LP & $\mathrm{p}$ \\
\hline Uso de alfa 2 agonistas S/N (\%) & $87 / 13$ & $64 / 36$ & $67 / 33$ & $80 / 20$ & 0.52 \\
\hline Tipo de anestesia GB/E (\%) & $75 / 25$ & $79 / 21$ & $87 / 13$ & $73 / 27$ & 0.81 \\
\hline Vol. Ilenado globo (mL) & $4(3-4)$ & $4(3-5)$ & $5(3.75-5.25)$ & $5(3.75-5.25)$ & 0.21 \\
\hline Fentanilo $(\mu \mathrm{g})$ & $480(357.5-592.5)$ & $512.5(450-600)$ & $400(325-450)$ & $350(300-565)$ & 0.18 \\
\hline Duración anestesia (min) & $117.5(87.5-156.25)$ & $135(115-180)$ & $115(90-142.5)$ & $110(90-210)$ & 0.38 \\
\hline Duración cirugía (min) & $85(67.5-120)$ & $105(90-135)$ & $91.5(67.5-120)$ & $107.5(63.75-172.5)$ & 0.30 \\
\hline Fin de la anestesia y extubación (min) & $7.5(3.75-12.75)$ & $7(5-10)$ & $7(6-12.75)$ & $8.5(6.75-9.25)$ & 0.81 \\
\hline Tos al retirar TE N/S (\%) & $81 / 19$ & $77 / 23$ & $67 / 33$ & $86 / 14$ & 0.7 \\
\hline Uso de sonda orogástrica N/S (\%) & $50 / 50$ & $50 / 50$ & $47 / 53$ & $71 / 29$ & 0.84 \\
\hline \multicolumn{6}{|l|}{ Analgesia postoperatoria } \\
\hline Uso de opioides N/T/M/MT/B (\%) & $56 / 0 / 32 / 6 / 6$ & $38 / 0 / 31 / 23 / 8$ & $47 / 7 / 46 / 0 / 0$ & $57 / 7 / 29 / 0 / 7$ & 0.56 \\
\hline Uso de AINEs N/P/KET/K/M (\%) & $6 / 94 / 0 / 0 / 0$ & 8/76/8/0/8 & 0/80/0/7/13 & 0/79/0/7/14 & 0.38 \\
\hline
\end{tabular}


Figura 1: Frecuencia de dolor laringotraqueal durante las primeras 24 horas del postoperatorio.

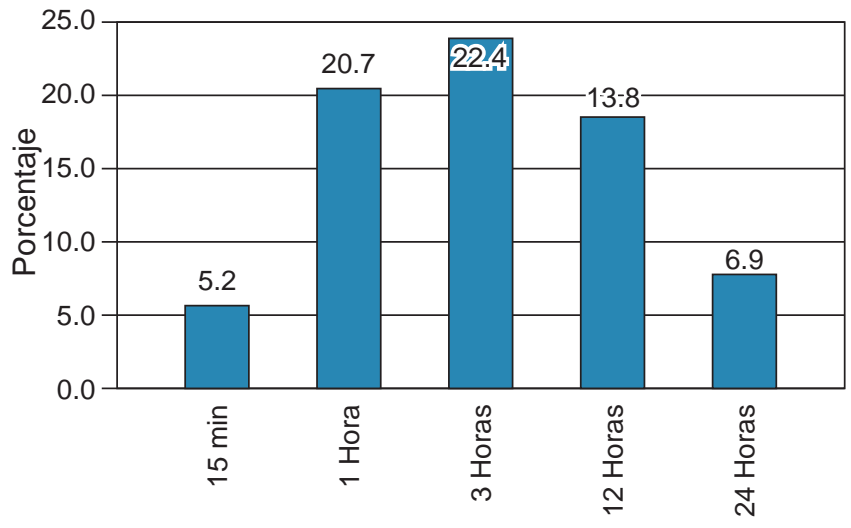

Figura 2: Frecuencia de dolor laringotraqueal ENA $>3$ durante las primeras 24 horas del postoperatorio.

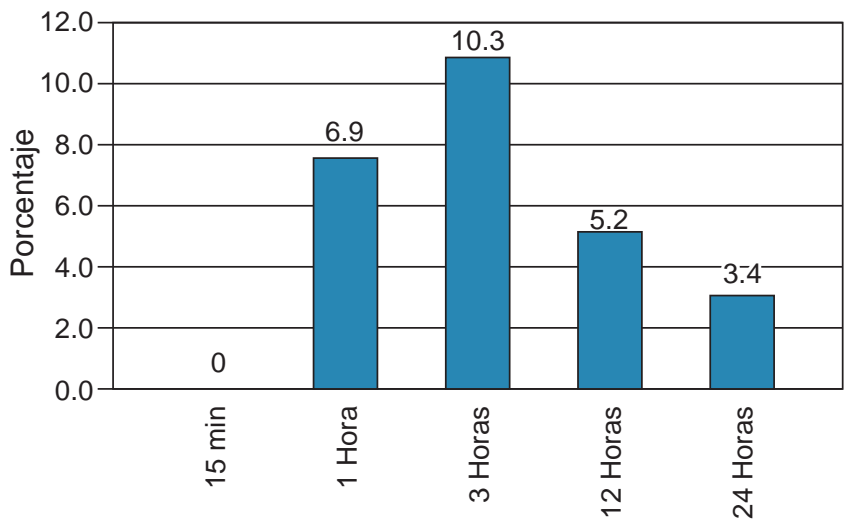

cualquier intensidad de dolor y sólo de $10.3 \%$ con una intensidad ENA $>3$.

Una maniobra frecuente en nuestro medio es el uso de gel o aerosol de lidocaína directamente en la orofaringe al realizar la laringoscopia o en el exterior del globo del tubo endotraqueal, pero en diversos estudios su uso aumentaba de manera significativa la incidencia de dolor laringotraqueal. $3,7,10,12,13$ Esto posiblemente debido a los aditivos que contienen el aerosol y el gel de lidocaína (mentol y etanol) que pudieran provocar un efecto irritante local 7,13 o una adherencia del tubo a la mucosa traqueal causando desgarro del epitelio al retirar el tubo, 2,4,5 por lo que no incluimos esta técnica en nuestro estudio.

La lidocaína alcalinizada infundida dentro del globo del tubo endotraqueal favorece su difusión a través del globo del tubo endotraqueal. 2,14,15 $\mathrm{Al}$ aumentar el pH de la solución anestésica se incrementa la fracción no ionizada de la lidocaína y por lo tanto, aumenta la tasa de difusión hasta 73 veces, ${ }^{14}$ ya que el globo de los tubos endotraqueales está hecho de un material semipermeable. Camacho Navarro y colaboradores observaron que la concentración plasmática se mantenía en $1.12 \mu \mathrm{g} / \mathrm{mL}$ desde los primeros 10 minutos y hasta las tres horas con dosis de promedio de $123.5 \mathrm{mg}$ de lidocaína, ${ }^{2,15}$ por lo que se considera una intervención segura. Camacho Navarro y su equipo y Estebe y colegas también demostraron que la lidocaína alcalinizada dentro del globo del tubo endotraqueal es superior al placebo en la disminución de la incidencia de dolor laringotraqueal ( $p=0.02$ y $p<0.0001$ respectivamente).

La bencidamina también se utiliza en aerosol aplicada por fuera del tubo endotraqueal. La bencidamina es un analgésico y antiinflamatorio no esteroideo con poca absorción sistémica. En 2010, Huang y colaboradores publicaron la efectividad de bencidamina en aerosol por fuera del tubo para este mismo propósito mostrando superioridad al placebo con un RR 0.36 (IC 0.21-0.60 y p $<0.05)$, mientras que Huang y su equipo detectaron una disminución en la incidencia con bencidamina en aerosol en comparación con lidocaína no alcalinizada por dentro del globo a diferentes concentraciones y placebo con una $\mathrm{p}<0.05$.

Por estos antecedentes, al diseñar el estudio esperábamos encontrar una diferencia estadísticamente significativa del grupo LB contra los demás grupos.

Existe la posibilidad de que el clorhidrato de bencidamina, al ser una base débil y estar alcalinizada la lidocaína se alcance el umbral de efectividad de la alcalinización al utilizar ambos fármacos de manera conjunta y por ello, resulte sólo ligeramente mejor que el control. Existe también la posibilidad de una reacción fisicoquímica entre ambos fármacos que los inactive al estar en contacto uno con otro. La lidocaína alcalinizada por dentro del globo del tubo endotraqueal mostró una diferencia estadísticamente

Figura 3: Dolor laringotraqueal durante las primeras 24 horas del postoperatorio según el grupo experimental.

$\mathrm{PP}=$ grupo placebo-placebo; $\mathrm{LB}=$ grupo lidocaína-bencidamina; PB = grupo placebo-bencidamina; LP = grupo lidocaína-placebo.

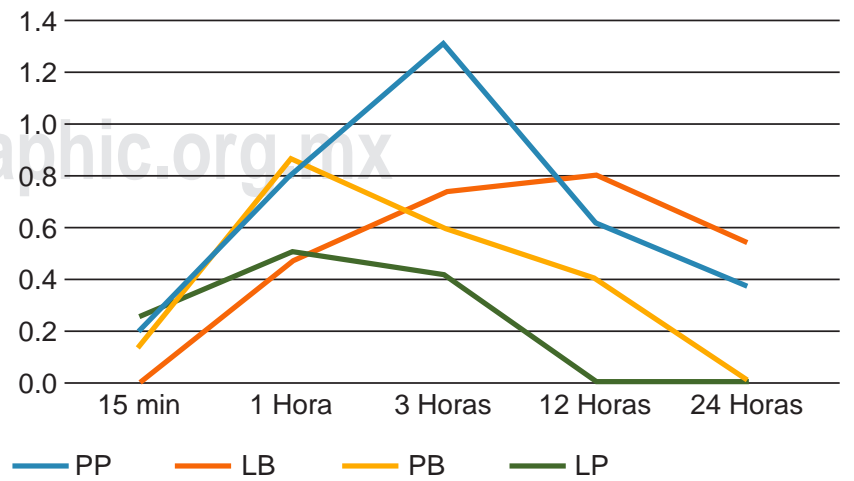


significativa con respecto a los demás fármacos para disminuir la frecuencia e intensidad de dolor laringotraqueal postoperatorio, principalmente a las $3 \mathrm{~h}, 12 \mathrm{~h}$ y $24 \mathrm{~h}$.

En nuestro medio, observamos que el costo de aplicar bencidamina por fuera del tubo endotraqueal y la instilación de lidocaína alcalinizada por dentro del globo del tubo endotraqueal es muy bajo, aun cuando varía entre instituciones privadas, lo cual nos permite realizar estas maniobras sin causar un gasto excesivo para un efecto adverso poco común y en la mayoría de los casos, de baja intensidad. Cabe mencionar que la analgesia postoperatoria en casi todos los pacientes fue multimodal, incluyendo paracetamol, un AINE y un opioide, lo cual deja poco lugar para que el dolor laringotraqueal postoperatorio sea intenso.

Como alternativa aceptable, encontramos la utilización de bencidamina en aerosol por fuera del tubo endotraqueal para un efecto menos intenso, pero igualmente eficaz. Es importante recalcar que ambos fármacos por sí solos mantuvieron el dolor en ENA $<3$ en todos los momentos en más de $75 \%$ de los pacientes, por lo que cualquiera de los dos es una opción segura y costo-efectiva para reducir la intensidad del dolor laringotraqueal postoperatorio.

Consideramos que la principal limitante del estudio es que al no conocer la intensidad real del dolor laringotraqueal, ya que ninguno de los estudios publicados lo menciona, y ser desde el punto de vista clínico una intensidad tan baja (dolor con mediana de ENA menor de 2), no se pudo evaluar la eficacia de los distintos tratamientos propuestos en los casos de dolor laringotraqueal intenso (ENA mayor de 4). Una línea de investigación adicional en el futuro sería identificar la frecuencia de dolor laringotraqueal intenso y los factores de riesgo de presentarla para evaluar la eficacia de la lidocaína alcalinizada en esos casos.

\section{REFERENCIAS}

1. Huang YS, Hung NK, Lee MS, Kuo CP, Yu JC, Huang GS et al. The effectiveness of benzydamine hydrochloride spraying on the endotracheal tube cuff or oral mucosa for postoperative sore throat. Anesth Analg. 2010; 111 (4): 887-891.

2. Navarro LH, Lima RM, Aguiar AS, Braz JR, Carness JM, Módolo NS. The effect of intracuff alkalinized $2 \%$ lidocaine on emergence coughing, sore throat, and hoarseness in smokers. Rev Assoc Med Bras (1992). 2012; 58 (2): 248-253.

3. McHardy FE, Chung F. Postoperative sore throat: cause, prevention and treatment. Anaesthesia. 1999; 54 (5): 444-453.
4. Lam F, Lin YC, Tsai HC, Chen TL, Tam KW, Chen CY. Effect of intracuff lidocaine on postoperative sore throat and the emergence phenomenon: a systematic review and meta-analysis of randomized controlled trials. PLoS One. 2015; 10 (8): e0136184.

5. Estebe JP, Gentili M, Le Corre P, Dollo G, Chevanne F, Ecoffey C. Alkalinization of intracuff lidocaine: efficacy and safety. Anesth Analg. 2005; 101 (5): 1536-1541.

6. D'Aragon F, Beaudet N, Gagnon V, Martin R, Sansoucy Y. The effects of lidocaine spray and intracuff alkalinized lidocaine on the occurrence of cough at extubation: a double-blind randomized controlled trial. Can J Anaesth. 2013; 60 (4): 370376.

7. Hung NK, Wu CT, Chan SM, Lu CH, Huang YS, Yeh CC et al. Effect on postoperative sore throat of spraying the endotracheal tube cuff with benzydamine hydrochloride, $10 \%$ lidocaine, and $2 \%$ lidocaine. Anesth Analg. 2010; 111 (4): 882-886.

8. Jaensson M, Gupta A, Nilsson UG. Risk factors for development of postoperative sore throat and hoarseness after endotracheal intubation in women: a secondary analysis. AANA J. 2012; 80 (4 Suppl): S67-S73.

9. Biro P, Seifert B, Pasch T. Complaints of sore throat after tracheal intubation: a prospective evaluation. Eur J Anaesthesiol. 2005; 22 (4): 307-311.

10. Chang JE, Min SW, Kim CS, Han SH, Kwon YS, Hwang JY. Effect of prophylactic benzydamine hydrochloride on postoperative sore throat and hoarseness after tracheal intubation using a double-lumen endobronchial tube: a randomized controlled trial. Can J Anaesth. 2015; 62 (10): 1097-1103.

11. Jaensson M, Gupta A, Nilsson U. Gender differences in sore throat and hoarseness following endotracheal tube or laryngeal mask airway: a prospective study. BMC Anesthesiol. 2014; 14: 56.

12. El-Boghdadly K, Bailey CR, Wiles MD. Postoperative sore throat: a systematic review. Anaesthesia. 2016; 71 (6): 706-717.

13. Estebe JP, Delahaye S, Le Corre P, Dollo G, Le Naoures A, Chevanne $F$ et al. Alkalinization of intra-cuff lidocaine and use of gel lubrication protect against tracheal tube-induced emergence phenomena. $\mathrm{Br} J$ Anaesth. 2004; 92 (3): 361-366.

14. Huang CJ, Tsai MC, Chen CT, Cheng CR, Wu KH, Wei TT. In vitro diffusion of lidocaine across endotracheal tube cuffs. Can J Anaesth. 1999; 46 (1): 82-86.

15. Navarro LH, Braz JR, Nakamura G, Lima RM, Silva Fde P, Módolo NS. Effectiveness and safety of endotracheal tube cuffs filled with air versus filled with alkalinized lidocaine: a randomized clinical trial. Sao Paulo Med J. 2007; 125 (6): 322-328.

16. Aqil M, Khan MU, Mansoor S, Mansoor S, Khokhar RS, Narejo AS. Incidence and severity of postoperative sore throat: a randomized comparison of Glidescope with Macintosh laryngoscope. BMC Anesthesiol. 2017; 17 (1): 127.

17. Macario A, Weinger M, Carney S, Kim A. Which clinical anesthesia outcomes are important to avoid? The perspective of patients. Anesth Analg. 1999; 89 (3): 652-658.

18. Wilson ME, Apiegelhalter D, Robertson JA et al. Predicting difficult intubation. Br J Anesth. 1988; 61: 211-216. 